\title{
THE KERNEL OF THE EQUIVARIANT KIRWAN MAP AND THE RESIDUE FORMULA
}

\author{
Lisa C. Jefrrey and Augustin-Liviu Mare
}

\begin{abstract}
A BSTRACT. Using the notion of equivariant Kirwan map, as defined by Goldin [Go], we prove that - in the case of Hamiltonian torus actions with isolated fixed points - Tolman and Weitsman's description of the kernel of the Kirwan map can be deduced directly from the residue theorem of [Je-Ki1] and [Je-Ki2]. A characterization of the kernel of the Kirwan map in terms of residues of one variable (i.e. associated to Hamiltonian circle actions) is obtained.
\end{abstract}

\section{$\S 1$ INTRODUCTION}

Let $H$ be a torus acting in a Hamiltonian fashion on a compact symplectic manifold $N$ and $S \subset H$ a circular subgroup. Assume that $0 \in \mathfrak{s}^{*}$ is a regular value of the moment map $\mu_{S}: M \rightarrow \mathfrak{s}^{*}$ which corresponds to the $S$ action on $N$. Define the symplectic reduction $N_{\text {red }}=\mu_{S}^{-1}(0) / S$ and consider

$$
\kappa_{S}: H_{H}^{*}(N) \rightarrow H_{H}^{*}\left(\mu_{S}^{-1}(0)\right)=H_{H / S}^{*}\left(N_{\text {red }}\right) .
$$

R. Goldin [Go] called this map the equivariant Kirwan map and then she proved:

Theorem 1.1. (see [Go, Theorem 1.2]) The map $\kappa_{S}$ is surjective.

Assume that the fixed point set $\mathcal{F}=N^{S}$ consists of isolated points. Consider

$$
\mathcal{F}_{+}=\left\{F \in \mathcal{F} \mid \mu_{S}(F)>0\right\}
$$

and

$$
\mathcal{F}_{-}=\left\{F \in \mathcal{F} \mid \mu_{S}(F)<0\right\} .
$$

Let $X, Y_{1}, \ldots, Y_{m}$ be variables corresponding to an integral basis of $\mathfrak{h}^{*}$ such that

$\left.X\right|_{\mathfrak{s}}$ corresponds to an integral basis of $\mathfrak{s}^{*}$ and $\left.Y_{j}\right|_{\mathfrak{s}}=0, j \geq 1$.

We will identify

$$
H_{H}^{*}(\mathrm{pt})=S\left(\mathfrak{h}^{*}\right)=\mathbb{C}\left[X, Y_{1}, \ldots, Y_{m}\right] .
$$

The following residue formula has been proved in [Je-Ki1] and [Je-Ki2] (see also [Ka], [Gu-Ka]): 
Theorem 1.2. For any $\eta \in H_{H}^{*}(N)$ we have ${ }^{1}$

$$
\kappa_{S}(\eta)\left[N_{\text {red }}\right]=c \sum_{F \in \mathcal{F}_{+}} \operatorname{Res}_{X}^{+} \frac{\left.\eta\right|_{F}}{e_{F}} .
$$

Here $e_{F} \in H_{H}^{*}(F)$ denotes the $H$-equivariant Euler class of the normal bundle $\nu(F), c$ is a non-zero constant ${ }^{2}$, and $\operatorname{Res}_{X}^{+}$is defined below in (1.3).

According to [Je-Ki2, Definition 3.3 and Proposition 3.4], the value of $\operatorname{Res}_{X}^{+}$on a rational function of the type

$$
h\left(X, Y_{1}, \ldots, Y_{m}\right)=\frac{p\left(X, Y_{1}, \ldots, Y_{m}\right)}{\prod_{k}\left(m_{k} X+\sum_{i} \beta_{k i} Y_{i}\right)}
$$

where $p \in \mathbb{C}\left[X,\left\{Y_{i}\right\}\right], m_{i} \in \mathbb{Z} \backslash\{0\}$ and $\beta_{k i} \in \mathbb{C}$, can be obtained as follows: regard $Y_{1}, \ldots, Y_{m}$ as constants (i.e. as complex numbers) and set

$$
\operatorname{Res}_{X}^{+}(h)=\sum_{b \in \mathbb{C}} \operatorname{Res}_{X=b} \frac{p}{q}
$$

where on the right hand side $\frac{p}{q}$ is interpreted as a meromorphic function in the variable $X$ on $\mathbb{C}$. It remains to note that only residues of expressions of the type (1.2) are involved in (1.1). For we may assume that each normal bundle $\nu(F), F \in \mathcal{F}_{+}$, is a direct sum of $H$-invariant line bundles and in this way the Euler class $e_{F}$ is the product of $H$-equivariant first Chern classes of those line bundles.

Remark 1.3. For future reference, we mention that

$$
\operatorname{Res}_{X}^{+} \frac{1}{X+\sum_{i} \beta_{i} Y_{i}}=1
$$

Remark 1.4. Guillemin and Kalkman gave another definition of the residue of $h$ given by (1.2) (see [Gu-Ka, section 3]). Denote $\beta_{k}(Y)=\sum_{i} \beta_{k i} Y_{i}$ and write

$$
\frac{1}{\prod_{k}\left(m_{k} X+\beta_{k}(Y)\right)}=\frac{1}{\prod_{k} m_{k} X\left(1+\frac{\beta_{k}(Y)}{m_{k} X}\right)}=\prod_{k}\left(m_{k} X\right)^{-1} \prod_{k}\left(1-\frac{\beta_{k}(Y)}{m_{k} X}+\left(\frac{\beta_{k}(Y)}{m_{k} X}\right)^{2}-\ldots\right) .
$$

Multiply the right hand side by the polynomial $p$, and add together all coefficients of $X^{-1}$ : the result is the Guillemin-Kalkman residue. It is a simple exercise to show that the latter coincides with the residue $\operatorname{Res}_{X}^{+}$of [Je-Ki2] (one uses the fact that the sum of the residues of the 1-form $h(X) d X$ on the Riemann sphere equals zero).

Since $\kappa_{S}$ is a ring homomorphism, from Theorem 1.2 we deduce:

\footnotetext{
${ }^{1}$ Note that both sides of the equation are in $\mathbb{C}\left[Y_{1}, \ldots, Y_{m}\right]$.

${ }^{2}$ The exact value of $c$ is not needed in our paper, but the interested reader can find it in [Je-Ki2, §3].
} 
Corollary 1.5.

$$
\kappa_{S}(\eta) \kappa_{S}(\zeta)\left[N_{\text {red }}\right]=\sum_{F \in \mathcal{F}_{+}} \operatorname{Res}_{X}^{+} \frac{\left.(\eta \zeta)\right|_{F}}{e_{F}}
$$

Since $0 \in \mathfrak{s}^{*}$ is a regular value of $\mu_{S}, S$ acts on $\mu_{S}^{-1}(0)$ with finite stabilizers. Hence $\mu_{S}^{-1}(0) / S$ has at worst orbifold singularities and in particular it satisfies Poincaré duality. From Corollary 1.5 it follows that $\eta \in H_{H}^{*}(N)$ is in ker $\kappa_{S}$ iff

$$
\sum_{F \in \mathcal{F}_{+}} \operatorname{Res}_{X}^{+} \frac{\left.(\eta \zeta)\right|_{F}}{e_{F}}=0
$$

for all $\zeta \in H_{H}^{*}(N)$.

The main goal of our paper is to compare the latter description of ker $\kappa_{S}$ to the one given by Goldin in [Go]. We will assume that the fixed point sets $N^{S}$ and $N^{H}$ are equal, which holds for a generic choice of the subgroup $S$ of $H$ (see Remark 1.9 below).

Theorem 1.6. (see [Go, Theorem 1.5]) We have

$$
\operatorname{ker} \kappa_{S}=K_{-} \oplus K_{+}
$$

where $K_{-}$is the set of all elements of $H_{H}^{*}(N)$ vanishing on all components $F \in \mathcal{F}_{-}$, and similarly for $K_{+}$.

The goal of our paper is to give a direct proof of the following result:

Theorem 1.7. Assume that the fixed point set $N^{H}=N^{S}$ is finite. A class $\eta \in H_{H}^{*}(N)$ satisfies (1.4) for all $\zeta \in H_{H}^{*}(N)$ if and only if $\eta \in K_{+} \oplus K_{-}$.

It is obvious that any $\eta \in K_{+}$satisfies (1.4). On the other hand, by the Atiyah-BottBerline-Vergne localization formula (see [At-Bo], [Be-Ve]) the sum

$$
\sum_{F \in \mathcal{F}} \frac{\left.(\eta \zeta)\right|_{F}}{e_{F}}
$$

is a polynomial in $X,\left\{Y_{i}\right\}$, so that its residue is zero. It follows that any $\eta$ in $K_{-}$satisfies (1.4) as well. The hard part will be to prove that if $\eta$ satisfies (1.4) for any $\zeta \in H_{H}^{*}(N)$, then $\eta$ is in $K_{-} \oplus K_{+}$.

Remark 1.8. Theorem 1.7 is a generalization of the main result of [Je].

Remark 1.9. Let $M$ be a symplectic manifold acted on by a torus $G$ in a Hamiltonian fashion such that the fixed point set $M^{G}$ is finite and let $\mu_{G}: M \rightarrow \mathfrak{g}^{*}$ be the corresponding 
moment map. Suppose that $0 \in \mathfrak{g}^{*}$ is a regular value of $\mu_{G}$ and consider the corresponding symplectic reduction

$$
M / / G=\mu_{G}^{-1}(0) / G
$$

as well as the Kirwan surjection

$$
\kappa: H_{G}^{*}(M) \rightarrow H^{*}(M / / G) .
$$

Using reduction in stages, results like Theorem 1.6 or Theorem 1.7 can be used in order to obtain descriptions of ker $\kappa$. The following construction is needed: There exists a "generic" (in the sense of [Go, section 1]) circle $S_{1} \subset G$ with Lie algebra $\mathfrak{s}_{1}$ such that

- $M^{S_{1}}=M^{G}$

- 0 is a regular value of the moment map $\mu_{S_{1}}: M \rightarrow \mathfrak{s}_{1}^{*}$.

Consider the action of $G / S_{1}$ on the reduced space $M / / S_{1}=\mu_{S_{1}}^{-1}(0) / S_{1}$. As before, there exists a generic circle $S_{2} \subset G / S_{1}$ such that

- $\left(M / / S_{1}\right)^{S_{2}}=\left(M / / S_{1}\right)^{G / S_{1}}$

- 0 is a regular value of the moment map $\mu_{S_{2}}: M / / S_{1} \rightarrow \mathfrak{s}_{2}^{*}$.

The equivariant Kirwan map corresponding to the subtorus $S_{1} \times S_{2}$ of $G$ is the following composition of maps:

$$
H_{G}^{*}(M) \stackrel{\kappa_{1}}{\longrightarrow} H_{G / S_{1}}^{*}\left(M / / S_{1}\right) \stackrel{\kappa_{2}}{\longrightarrow} H_{G /\left(S_{1} \times S_{2}\right)}^{*}\left(M / /\left(S_{1} \times S_{2}\right)\right),
$$

where we have used "reduction in stages". In fact the procedure can be continued giving rise to a sequence of tori

$$
\{1\}=T_{0} \subset T_{1} \subset T_{2} \subset \ldots \subset T_{m}=G
$$

where $T_{1}=S_{1}, T_{2}=S_{1} \times S_{2}, \ldots$ with the following properties:

- $\left(M / / T_{j-1}\right)^{T_{j} / T_{j-1}}=\left(M / / T_{j-1}\right)^{G / T_{j-1}}$

- 0 is a regular value of the moment map $\mu_{T_{j} / T_{j-1}}$ on $M / / T_{j-1}$.

By reduction in stages, the Kirwan map $\kappa$ decomposes as

$$
\kappa=\kappa_{m} \circ \ldots \circ \kappa_{1}
$$

where

$$
\kappa_{j}=\kappa_{T_{j} / T_{j-1}}: H_{G / T_{j-1}}^{*}\left(M / / T_{j-1}\right) \rightarrow H_{G / T_{j}}^{*}\left(M / / T_{j}\right)
$$

is the $T_{j} / T_{j-1}$-equivariant Kirwan map. Goldin [Go] used this decomposition in order to deduce from Theorem 1.6 the Tolman-Weitsman description of ker $\kappa$ (see [To-We]). Theorem 1.7 of our paper — with $N=M / / T_{j-1}, H=G / T_{j-1}$ and $S=T_{j} / T_{j-1}$ shows that the same Tolman-Weitsman description of ker $\kappa$ is equivalent to the obvious characterization in terms of residues arising from the components $\kappa_{j}$ of $\kappa$. 


\section{$\S 2$ EXPRESSING ker $\kappa_{S}$ IN TERMS OF RESIDUES}

We will give a proof of Theorem 1.7. Take $\xi \in \mathfrak{s}$ a non-zero vector and $f=\mu_{S}^{\xi}=$ $\left\langle\mu_{S}, \xi\right\rangle: N \rightarrow \mathbb{R}$ the function induced on $N$ by the moment map $\mu_{S}: N \rightarrow \mathfrak{s}^{*}$. This is an $H$-equivariant Morse function, whose critical set is $N^{S}=N^{H}$. For any $F \in \mathcal{F}$, we have the $H$-equivariant splitting of the tangent space

$$
T_{F} N=\nu_{f}^{-}(F) \oplus \nu_{f}^{+}(F)
$$

determined by the sign of the Hessian on the two summands. Let $e\left(\nu_{f}^{-}(F)\right), e\left(\nu_{f}^{+}(F)\right) \in$ $H_{H}^{*}(F)$ be the corresponding $H$-equivariant Euler classes. The following two results can be proved by using Morse theory (see for example [Go]):

Proposition 2.1. Suppose $\eta \in H_{H}^{*}(N)$ restricts to zero on all $G \in \mathcal{F}$ for which $f(G)<$ $f(F)$. Then $\left.\eta\right|_{F}$ is some $H_{H}^{*}(\mathrm{pt})$-multiple of $e\left(\nu_{f}^{-} F\right)$.

Proposition 2.2. For any $F \in \mathcal{F}$ there exists a class $\alpha^{-}(F) \in H_{H}^{*}(N)$ with the following properties:

1. $\left.\alpha^{-}(F)\right|_{G}=0$, for any $G \in \mathcal{F}$ which cannot be joined to $F$ along a sequence of integral lines of the negative gradient field $-\nabla f$ (in particular, for any $G \in \mathcal{F}$ with $f(G)<f(F)$ )

2. $\left.\alpha^{-}(F)\right|_{F}=e\left(\nu_{f}^{-} F\right)$.

In the same way there exists $\alpha^{+}(F) \in H_{H}^{*}(N)$ such that:

1. $\left.\alpha^{+}(F)\right|_{G}=0$, for any $G \in \mathcal{F}$ which cannot be joined to $F$ along a sequence of integral lines of the gradient field $\nabla f$ (in particular, for any $G \in \mathcal{F}$ with $f(G)>f(F)$ )

2. $\left.\alpha^{+}(F)\right|_{F}=e\left(\nu_{f}^{+} F\right)$.

From the injectivity theorem of Kirwan — which says that $\alpha \in H_{H}^{*}(N)$ is uniquely determined by its restrictions to $N^{H}$ — we deduce:

Corollary 2.3. The set $\left\{\alpha^{-}(F) \mid F \in \mathcal{F}\right\}$ is a basis of $H_{H}^{*}(N)$ as a $H_{H}^{*}(p t)$-module.

Consider the space $\hat{H}_{H}^{*}(N)$ consisting of all expressions of the type

$$
\sum_{F \in \mathcal{F}} r_{F} \alpha^{-}(F)
$$

where $r_{F}$ is in the ring $\mathbb{C}\left(X,\left\{Y_{i}\right\}\right)$ of rational expressions in $X,\left\{Y_{i}\right\}$ (i.e. quotients $p / q$, with $\left.p, q \in \mathbb{C}\left[X,\left\{Y_{i}\right\}\right], q \neq 0\right)$. The space $\hat{H}_{H}^{*}(N)$ is obviously a $\mathbb{C}\left(X,\left\{Y_{i}\right\}\right)$-algebra. 
Lemma 2.4. Take $\eta \in H_{H}^{*}(N)$ of degree $d$. Then we can decompose

$$
\eta=\eta_{+}+\eta_{-}
$$

where $\eta_{+}, \eta_{-} \in \hat{H}_{H}^{*}(N)$, such that

(i) $\eta_{+}\left|\mathcal{F}_{-}=0, \eta_{-}\right| \mathcal{F}_{+}=0$;

(ii) $\eta_{+}$and $\eta_{-}$are linear combinations of $\alpha^{-}(F), F \in \mathcal{F}$, where the coefficients $r_{F}$ are rational functions whose denominators can be decomposed as products of linear factors of the type

$$
X+\sum_{i} \beta_{i} Y_{i}, \quad \beta_{i} \in \mathbb{C}
$$

Proof. By Corollary 2.3, we have

$$
\eta=\sum_{F \in \mathcal{F}} p_{F} \alpha^{-}(F)
$$

where $p_{F} \in \mathbb{R}\left[X,\left\{Y_{i}\right\}\right]$ are homogeneous polynomials. Take

$$
\tilde{\eta}_{-}=\sum_{F \in \mathcal{F}_{-}} p_{F} \alpha^{-}(F), \quad \tilde{\eta}_{+}=\sum_{F \in \mathcal{F}_{+}} p_{F} \alpha^{-}(F)
$$

By Proposition 2.2, $\tilde{\eta}_{+}$restricts to 0 on all $G \in \mathcal{F}_{-}$.

Consider the ordering $F_{1}, F_{2}, \ldots$ of the elements of $\mathcal{F}_{+}$such that

$$
0<f\left(F_{1}\right)<f\left(F_{2}\right)<\ldots
$$

There exists a rational function $r_{1}\left(X,\left\{Y_{i}\right\}\right) \in \mathbb{C}\left(X,\left\{Y_{i}\right\}\right)$ such that

$$
\left.\tilde{\eta}_{-}\right|_{F_{1}}=r_{1}\left(X,\left\{Y_{i}\right\}\right) e\left(\nu_{f}^{-} F_{1}\right)
$$

which means that the form

$$
\tilde{\eta}_{1}:=\tilde{\eta}_{-}-r_{1}\left(X,\left\{Y_{i}\right\}\right) \alpha^{-}\left(F_{1}\right)
$$

vanishes at $F_{1}$. There exists another rational function $r_{2}\left(X,\left\{Y_{i}\right\}\right) \in \mathbb{C}\left(X,\left\{Y_{i}\right\}\right)$, with the property that

$$
\left.\tilde{\eta}_{1}\right|_{F_{2}}=r_{2}\left(X,\left\{Y_{i}\right\}\right) e\left(\nu_{f}^{-} F_{2}\right)
$$

which implies that the form

$$
\tilde{\eta}_{1}-r_{2}\left(X,\left\{Y_{i}\right\}\right) \alpha^{-}\left(F_{2}\right)
$$


vanishes at both $F_{1}$ and $F_{2}$. We continue this process and we get the decomposition claimed in the proposition as follows:

$$
\eta_{-}=\tilde{\eta}_{-}-r_{1} \alpha^{-}\left(F_{1}\right)-r_{2} \alpha^{-}\left(F_{2}\right)-\ldots, \quad \eta_{+}=\tilde{\eta}_{+}+r_{1} \alpha^{-}\left(F_{1}\right)+r_{2} \alpha^{-}\left(F_{2}\right)+\ldots
$$

Property (ii) follows from the fact that for each $F \in \mathcal{F}_{+}$, the weights of the representation of $S$ on $\nu_{f}^{-}(F)$ are all non-zero.

We are now ready to prove the main result of the paper.

Proof of Theorem 1.7. Take $\eta \in H_{H}^{d}(N)$ satisfying

$$
\operatorname{Res}_{X}^{+} \sum_{F \in \mathcal{F}_{+}} \frac{\left.(\eta \zeta)\right|_{F}}{e_{F}}=0
$$

for all $\zeta \in H_{H}^{*}(N)$. We consider the decomposition

$$
\eta=\eta_{-}+\eta_{+}
$$

with $\eta_{-}, \eta_{+} \in \hat{H}_{H}^{*}(N)$ given by Lemma 2.4. We show that $\eta_{-}$and $\eta_{+}$are actually in $H_{H}^{*}(N)$. More precisely, if $\eta_{+}$is of the form

$$
\eta_{+}=\sum_{G \in \mathcal{F}_{+}} \frac{p_{G}}{q_{G}} \alpha^{-}(G)
$$

with $p_{G}, q_{G} \in \mathbb{C}\left[X, Y_{i}\right]$ we show that

$$
q_{G} \text { divides } p_{G}
$$

for any $G \in \mathcal{F}_{+}$.

From (2.1) and the fact that $\eta_{-} \mid \mathcal{F}_{+}=0$, we deduce that

$$
\operatorname{Res}_{X}^{+} \sum_{F \in \mathcal{F}_{+}} \frac{\left.\left(\eta_{+} \zeta\right)\right|_{F}}{e_{F}}=0
$$

which is equivalent to

$$
\operatorname{Res}_{X}^{+} \sum_{F, G \in \mathcal{F}_{+}} \frac{p_{G}}{q_{G}} \cdot \frac{\left.\left.\alpha^{-}(G)\right|_{F} \zeta\right|_{F}}{e_{F}}=0
$$


for all $\zeta \in H_{H}^{*}(N)$. Consider again the ordering $F_{1}, F_{2}, \ldots$ of the elements of $\mathcal{F}_{+}$such that

$$
f\left(F_{1}\right)<f\left(F_{2}\right)<\ldots .
$$

We prove by induction on $k \geq 1$ that $q_{F_{k}}$ divides $p_{F_{k}}$.

In (2.2) we put $\zeta=p \alpha^{+}\left(F_{1}\right)$, where $p \in \mathbb{C}\left[X, Y_{i}\right]$ is an arbitrary polynomial. Since

$$
e\left(\nu_{f}^{-} F_{1}\right) e\left(\nu_{f}^{+} F_{1}\right)=e_{F_{1}},
$$

we deduce that

$$
\operatorname{Res}_{X}^{+} p \frac{p_{F_{1}}}{q_{F_{1}}}=0
$$

for any $p \in \mathbb{C}\left[X, Y_{1}, \ldots, Y_{m}\right]$ (we are using the fact that $\left.\alpha^{+}\left(F_{1}\right)\right|_{F}=0$ if $\mu(F)>\mu\left(F_{1}\right)$ while $\left.\alpha^{-}(G)\right|_{F}=0$ if $\mu(F)<\mu(G)$ : hence the only nonzero contribution comes from $F=G=F_{1}$ ). From Lemma 2.5 (see below) we deduce that

$$
q_{F_{1}} \text { divides } p_{F_{1}} \text {. }
$$

Now we fix $k \geq 2$, assume that $q_{F_{i}}$ divides $p_{F_{i}}$ for any $i<k$ and show that $q_{F_{k}}$ divides $p_{F_{k}}$. In $(2.2)$ put $\zeta=p \alpha^{+}\left(F_{k}\right)$, with $p \in \mathbb{C}\left[X, Y_{1}, \ldots, Y_{m}\right]$. We obtain

$$
\operatorname{Res}_{X}^{+} \sum_{i \leq j \leq k} p \frac{p_{F_{i}}}{q_{F_{i}}} \cdot \frac{\left.\left.\alpha^{-}\left(F_{i}\right)\right|_{F_{j}} \alpha^{+}\left(F_{k}\right)\right|_{F_{j}}}{e_{F_{j}}}=0 .
$$

The sum in the left hand side is over $i$ and $j$ ( $k$ being fixed). We divide into sums of the type

$$
\Sigma^{i}:=\operatorname{Res}_{X}^{+} \sum_{i \leq j \leq k} p \frac{p_{F_{i}}}{q_{F_{i}}} \cdot \frac{\left.\left.\alpha^{-}\left(F_{i}\right)\right|_{F_{j}} \alpha^{+}\left(F_{k}\right)\right|_{F_{j}}}{e_{F_{j}}}, \quad i \leq k .
$$

If $i<k$, then $\Sigma^{i}=0$, by the hypothesis that $q_{F_{i}}$ divides $p_{F_{i}}$ and the Atiyah-Bott-BerlineVergne localization formula for $p \frac{p_{F_{i}}}{q_{F_{i}}} \cdot \alpha^{-}\left(F_{i}\right) \alpha^{+}\left(F_{k}\right)$ (note that $\left.\left.\alpha^{-}\left(F_{i}\right)\right|_{F} \alpha^{+}\left(F_{k}\right)\right|_{F}=0$ for any $F$ which is not of the form $F_{j}$ with $\left.i \leq j \leq k\right)$.

Finally we show that $\Sigma^{k}=0$, which is equivalent to

$$
\operatorname{Res}_{X}^{+} p \frac{p_{F_{k}}}{q_{F_{k}}}=0
$$

for any $p \in \mathbb{C}\left[X, Y_{i}\right]$. By Lemma 2.5,

$$
q_{F_{k}} \text { divides } p_{F_{k}},
$$

which concludes the proof.

We have used the following result: 
Lemma 2.5. Let $f, g$ be in $\mathbb{C}\left[X,\left\{Y_{i}\right\}\right]$, where

$$
g=\prod_{k}\left(X+\sum_{i} \beta_{i k} Y_{i}\right)
$$

$\beta_{i k} \in \mathbb{C}$. If

$$
\operatorname{Res}_{X}^{+}\left(p \cdot \frac{f}{g}\right)=0
$$

for any $p \in \mathbb{C}\left[X,\left\{Y_{i}\right\}\right]$, then $g$ divides $f$.

Proof. Suppose that $g$ does not divide $f$. We can assume that $g$ and $f$ are relatively prime. Then there exist $p_{1}, p_{2} \in \mathbb{C}\left[X,\left\{Y_{i}\right\}\right]$ such that

$$
p_{1} f+p_{2} g=1
$$

From (2.3) it follows that

$$
\operatorname{Res}_{X}^{+} \frac{p}{g}=\operatorname{Res}_{X}^{+}\left(p p_{1} \cdot \frac{f}{g}+p_{2}\right)=0
$$

for any $p \in \mathbb{C}\left[X,\left\{Y_{i}\right\}\right]$. Now fix $k_{0}$ and set

$$
p=\prod_{k \neq k_{0}}\left(X+\sum_{i} \beta_{i k} Y_{i}\right) .
$$

We deduce that

$$
\operatorname{Res}_{X}^{+} \frac{1}{X+\sum_{i} \beta_{i k_{0}} Y_{i}}=0 .
$$

But the left hand side is actually 1 (see Remark 1.3), which is a contradiction.

\section{$\S 3$ Residues AND THE KERNEL OF THE KiRWAN MAP}

In this section we shall give a direct characterization of the kernel of the Kirwan map in terms of residues of one variable.

Let $M$ be a compact symplectic manifold equipped with a Hamiltonian $G$ action, where $G$ is a torus and let $\kappa: H_{G}^{*}(M) \rightarrow H^{*}(M / / G)$ be the Kirwan map. Consider the decomposition of $\kappa$ described in Remark 1.9 (see equation (1.5)). For each $j$ between 1 and $m$ we consider the commutative diagram 


$$
\begin{gathered}
H_{G / T_{j-1}}^{*}\left(M / / T_{j-1}\right) \stackrel{\kappa_{j}}{\longrightarrow} H_{G / T_{j}}^{*}\left(M / / T_{j}\right) \\
\downarrow \pi_{j} \quad \downarrow \pi \\
H_{T_{j} / T_{j-1}}^{*}\left(M / / T_{j-1}\right) \stackrel{\tilde{\kappa}_{j}}{\longrightarrow} H^{*}\left(M / / T_{j}\right)
\end{gathered}
$$

where $\tilde{\kappa}_{j}$ is the Kirwan map associated to the action of the circle $T_{j} / T_{j-1}$ on $M / / T_{j-1}$. For the same action we consider the moment map $\mu_{j}$, the fixed point set $\mathcal{F}^{j}=\left(M / / T_{j-1}\right)^{T_{j} / T_{j-1}}$ and the partition of the latter into $\mathcal{F}_{-}^{j}$ and $\mathcal{F}_{+}^{j}$ which consist of fixed points where $\mu_{j}$ is negative, respectively positive.

Definition 3.1. An element $\eta \in H_{T_{j} / T_{j-1}}^{*}\left(M / / T_{j-1}\right)$ is in $\mathrm{ker}_{\mathrm{res}, j}$ if

$$
\operatorname{Res}_{X_{j}=0} \sum_{F \in \mathcal{F}_{+}^{j}} \frac{\left.(\eta \zeta)\right|_{F}}{e_{F}\left(X_{j}\right)}=0
$$

for any $\eta \in H_{T_{j} / T_{j-1}}^{*}\left(M / / T_{j-1}\right)$, where $X_{j}$ is a variable corresponding to a basis of the dual of the Lie algebra of $T_{j} / T_{j-1}$ and Res $_{X_{j}=0}$ means the coefficient of $X_{j}^{-1}$.

For any $\alpha \in H_{G}^{*}(M)$ we define

$$
\alpha_{j}=\left(\kappa_{j} \circ \cdots \circ \kappa_{1}\right)(\alpha)
$$

$0 \leq j \leq m$. The goal of this section is to prove

Theorem 3.2. Let $\alpha \in H_{G}^{*}(M)$. Then $\kappa(\alpha)=0$ if and only if $\pi_{j}\left(\alpha_{j-1}\right) \in$ ker $_{\mathrm{res}, \mathrm{j}}$ for some $j \geq 1$.

Proof. If $\kappa(\alpha)=0$, by equation (1.5) there exists $j$ such that $\alpha_{j}=0$. Since $\alpha_{j}=\kappa_{j}\left(\alpha_{j-1}\right)$, we can use first the commutativity of the diagram (3.1) to deduce that $\tilde{\kappa}_{j}\left(\pi_{j} \alpha_{j-1}\right)=0$ and then the residue formula of [Je-Ki1] and [Je-Ki2] to deduce that $\pi_{j}\left(\alpha_{j-1}\right) \in \operatorname{ker}_{\text {res,j. }}$.

The opposite direction is less obvious: Take $\alpha \in H_{G}^{*}(M)$ of degree greater than zero such that $\pi_{j} \alpha_{j-1} \in \operatorname{ker}_{\mathrm{res}, \mathrm{j}}$. If $j=m$ then $\pi_{j}$ and $\pi$ are the identity maps, $\kappa_{j}=\tilde{\kappa}_{j}$ and we just have to apply the residue formula of [Je-Ki1] and [Je-Ki2] to deduce that $\kappa_{m}\left(\alpha_{m-1}\right)=\kappa(\alpha)=0$. If $j<m$, by the same residue formula, we have $\tilde{\kappa}_{j}\left(\pi_{j} \alpha_{j-1}\right)=$ 0 , which implies $\pi\left(\kappa_{j} \alpha_{j-1}\right)=0$. Note that $\operatorname{ker}(\pi)=H_{G / T_{j}}^{*}(\mathrm{pt})$ so $\pi\left(\kappa_{j} \alpha_{j-1}\right)=0$ is equivalent to $\kappa_{j} \alpha_{j-1} \in H_{G / T_{j}}^{*}(\mathrm{pt})$. The latter implies that $\left(\kappa_{m} \circ \cdots \circ \kappa_{j}\right) \alpha_{j-1}=0$ (since the map $\kappa_{m} \circ \cdots \circ \kappa_{j+1}$ sends all elements of $H_{G / T_{j}}^{*}(\mathrm{pt})$ of degree larger than zero to 0 , because the image of this map is $H^{*}(M / / G)$ and the image of the equivariant cohomology of a point under this map is the ordinary cohomology of a point). But this means $\kappa(\alpha)=0$ and the proof is now complete. 


\section{REFERENCES}

[At-Bo] M. F. Atiyah and R. Bott, The moment map and equivariant cohomology, Topology 23 (1984), $1-28$.

[Be-Ve] N. Berline and M. Vergne, Zéros d'un champ de vecteurs et classes caractéristiques équivariantes, Duke Math. J. 50 (1983), 539-549.

[Go] R. F. Goldin, An effective algorithm for the cohomology ring of symplectic reductions, Geom. Anal. Funct. Anal. 12 (2002), 567-583.

[Gu-Ka] V. Guillemin and J. Kalkman, The Jeffrey-Kirwan localization theorem and residue operations in equivariant cohomology, Jour. reine angew. Math. 470 (1996), 123-142.

[Je] L. C. Jeffrey, The residue formula and the Tolman-Weitsman theorem, preprint math.SG/0204051.

[Je-Ki1] L. C. Jeffrey and F. C. Kirwan, Localization for nonabelian group actions, Topology 34 (1995), 291-327.

[Je-Ki2] L. C. Jeffrey and F. C. Kirwan, Localization and the quantization conjecture, Topology 36 (1995), 647-693.

[Ka] J. Kalkman, Residues in nonabelian localization, preprint hep-th/9407115.

[To-We] S. Tolman and J. Weitsman, The cohomology ring of abelian symplectic quotients, preprint math.DG/9807173, to appear in Comm. Anal. Geom.

Mathematics Department

University of Toronto

Toronto, Ontario M5S $3 G 3$

Canada

jeffrey@math.toronto.edu

Mathematics Department

University of Toronto

Toronto, Ontario M5S $3 G 3$

Canada

amare@math . toronto. edu 